\title{
Laser-Induced Formation and Disintegration of Gold Nanopeanuts and Nanowires
}

\author{
Jung Shin Park, Jun Hee Yoon, Hyung Jun Kim, Young-Duk Huh, ${ }^{*}$ and Sangwoon Yoon*
Department of Chemistry, Center for Photofunctional Energy Materials, Dankook University, Yongin, Gyeonggi 448-701, Korea.*E-mail:ydhuh@dankook.ac.kr(Y.-D.Huh),sangwoon@dankook.ac.kr(S. Yoon) Received December 25, 2009, Accepted January 19, 2010

\begin{abstract}
We report the laser-induced formation of peanut-shaped gold nanoparticles (Au nanopeanuts) and gold nanowires (AuNWs), and their morphological properties. Pulsed laser irradiation of citrate-capped gold nanoparticles at $532 \mathrm{~nm}$ induces fragmentation, spherical growth, the formation of Au nanopeanuts, and the formation of AuNWs, sequentially. High-resolution transmission electron microscopy images reveal that the Au nanopeanuts are formed by instantaneous fusion of spherical nanoparticles in random orientation by laser heating. Furthermore, Au nanopeanuts are bridged in a linear direction to form AuNWs by an amorphous accumulation of gold atoms in the junction. The laser-produced Au nanopeanuts and AuNWs slowly disintegrate, restoring the spherical shape of the original Au nanoparticles when the laser irradiation is stopped. The addition of citrate effectively prevents them from transforming back to the nanospheres.
\end{abstract}

Key Words: Nanopeanuts, Nanowires, Photoreaction, Gold nanoparticles

\section{Introduction}

Noble metal nanoparticles (MNPs) have been of great importance in nanoscience for their unique material-, size- and shapedependent optical and electronic properties and broad applications. ${ }^{1-3}$ In particular, resonant excitation of the collective oscillation of conduction electrons in the MNPs, called surface plasmon resonance (SPR), gives rise to strong absorption. The molar absorption coefficient of gold nanoparticles (AuNPs) at the SPR wavelength, for example, is as large as $\sim 10^{10} \mathrm{M}^{-1} \mathrm{~cm}^{-1}, 5$ orders of magnitude greater than that of typical dye molecules. ${ }^{2}$ Strong absorption followed by the relaxation of the excited surface plasmons as heat makes it possible to exploit the AuNPs as specific cell-targeted photothermal therapeutic agents. ${ }^{4-8}$ Not only absorption but also scattering is greatly enhanced by the SPR particularly as the size of the MNPs increases. ${ }^{2}$ Biological imaging using enhanced contrast from the light scattering of AuNPs has been widely explored. ${ }^{9-11}$ Surface-enhanced Raman scattering (SERS) is another fascinating phenomenon resulting from SPR. ${ }^{12,13}$ Molecules closely located at or between MNPs experience greatly intensified electromagnetic fields induced by the SPR, producing a Raman signal enhanced by as much as $10^{14}$. Many areas of chemistry including single molecule probe and analytical sensors now take advantage of the great sensitivity of SERS. ${ }^{14-16}$

The SPR varies with the size and the shape of MNPs. ${ }^{2,3}$ Therefore, the capability to control the size and the shape of MNPs is of great significance. Generally, MNPs are synthesized by the reduction of precursor metal ions where the size and shape is determined by the surface energies of crystal planes and the interplay between stabilizing agents and the surfaces. ${ }^{17,18}$ Various shapes such as nanorods, nanoprisms, nanocubes, and nanocages have been synthesized by using the chemical methods. ${ }^{19-23}$ Alternative to such chemical approach, lasers may be used to produce nanocrystals or to control the size and shape of preformed nanoparticles. Lasers are particularly advantageous because they provide an intense burst of energy in a short period of time, which may allow one to produce a unique shape of nanoparticles that cannot be achieved in the conventional wet chemistry. Lasers also enable spatial control of nanoparticle production and manipulation, which can be utilized in multi-dimensional colorimetric patterning. ${ }^{24,25}$ In this article, we report the formation of peanut-shaped gold nanoparticles (Au nanopeanuts) and gold nanowires (AuNWs) by pulsed laser irradiation.

El-Sayed and coworkers transformed chemically synthesized gold nanorods into nanosphers by fs and ns laser pulses at 800 $\mathrm{nm}{ }^{26,27}$ Spherical AuNPs were fragmented to smaller spheres or further transformed to nanonetworks by pulsed laser irradiation at $532 \mathrm{~nm}$, depending on the concentration of surfactants (sodium dodecyl sulfate) or laser fluence. ${ }^{28-32}$ These laser-induced morpholgical changes are based upon interactions between the surface plasmons of MNPs and light. Resonantly excited surface plasmons decay via electron-electron, electron-phonon, and phonon-phonon interactions. ${ }^{33-35}$ The relaxation rate and pathway, and, consequently, the fate of the photoexcited nanoparticles are determined by the material properties of nanoparticles as well as the surrounding media (solvent, capping materials, etc.). Koda and coworkers suggested that the temperature of AuNPs rises to the near-boiling point due to the strong absorption of SPR at $532 \mathrm{~nm}$ and slow energy dissipation of the heated particles to solvent, resulting in the melting of AuNPs. ${ }^{28,32}$ Kleinermanns and coworkers investigated the influence of solvents on the photofragmentation rate of AuNPs. ${ }^{36}$

In this article, we report that irradiation of AuNPs induces the anisotropic growth of AuNPs to form Au nanopeanuts and AuNWs which are sustained while the irradiation continues, but which begin to slowly disintegrate into spherical nanoparticles when the irradiation stops.

\section{Material and Methods}

AuNP preparation, purification, and characterization. All chemical reagents were purchased from Aldrich and used as received. We synthesize AuNPs using the Turkevich method. ${ }^{37}$ 
Briefly, trisodium citrate $\left(50 \mathrm{~mL}, 3.4 \times 10^{-2} \mathrm{M}\right)$ is added to a heated $\mathrm{HAuCl}_{4}$ solution $\left(950 \mathrm{~mL}, 2.7 \times 10^{-4} \mathrm{M}\right)$ with vigorous stirring. The solution is further heated until the color changes from yellow to red. After cooling, the solution is kept in a brown bottle at $4{ }^{\circ} \mathrm{C}$. The solution contains not only the synthesized AuNPs stabilized by citrate but also an unknown amount of unreacted trisodium citrate and $\mathrm{HAuCl}_{4}$. To perform our experiments under quantitatively controlled conditions, we remove the residual reagents by centrifugation of the $10 \mathrm{~mL}$ solution at $10000 \mathrm{~g}(9960 \mathrm{rpm})$ for $10 \mathrm{~min}$. For reproducibility of the experiemnts, we use the same concentration of AuNPs in every set of experiments by adjucting the absorbance at $520 \mathrm{~nm}$ to $\sim 0.95$.

The prepared AuNPs are characterized using UV-visible absorption spectroscopy (Perkin-Elmer, Lambda 25) and high-resolution transmission electron microscopy (JEOL, JEM 3010). UV-visible absorption spectra show the strong surface plasmon band at $520 \mathrm{~nm}$, characteristic of gold nanospheres. Additional absorption bands at $253 \mathrm{~nm}$ and $<200 \mathrm{~nm}$ observed before centrifugation are attributed to absorption by the remaining $\mathrm{HAuCl}_{4}$ and citrate in solution, respectively (Supporting Information). Those bands disappear after centrifugation, indicating that the unreacted reagents are successfully removed. The size and the shape of AuNPs are measured by high-resolution transmission electron microscopy (HRTEM). The synthesized AuNPs were found to be uniform spherical particles with a diameter of $16 \pm$ $1 \mathrm{~nm}$.

Laser irradiation. The second harmonic generation of an $\mathrm{Nd}$ : YAG laser (Spectra-Physics, GCR-11) operating at $10 \mathrm{~Hz}$ produces $532 \mathrm{~nm}, 8 \mathrm{~ns}$ laser pulses, which are directed at a quartz cuvette containing $1.5 \mathrm{~mL}$ of AuNP solution. A laser beam with a diameter of $7 \mathrm{~mm}$ is used unfocused to illuminate the sample uniformly. The sample is irradiated by the laser pulses at 500 $\mathrm{mW}\left(130 \mathrm{~mJ} \cdot\right.$ puls $\left.^{-1} \cdot \mathrm{cm}^{-2}\right)$ while constantly being stirred. After a period of irradiation, the sample is measured for UV-visible absorption spectra. Furthermore, an aliquot of the sample is dropped on a carbon-coated copper grid and dried in the air to enable TEM measurements.

\section{Results and Discussion}

The formation of Au nanopeanuts and AuNWs by laser irradiation. Au nanopeanuts and AuNWs were obtained by the pulsed laser irradiation of citrate-stabilized Au nanospheres. We irradiated the synthesized Au nanospheres at $532 \mathrm{~nm}$ for a selected period of time and obtained UV-visible absorption spectra and HRTEM images to follow the structural evolution. Fig. 1 shows the change of the UV-visible absorption spectra as we increase the irradiation time. The spectral evolution can be divided into three stages according to the observed spectral features. For the first 3 min (Fig. 1a), the characteristic SPR band of the initially prepared AuNPs at $520 \mathrm{~nm}$ gradually shifts to $511 \mathrm{~nm}$ with its intensity decreasing. At the same time, we observe an increase of absorption in the UV region $(\sim 200 \mathrm{~nm})$. Continuing the irradiation further beyond $3 \mathrm{~min}$ shifts the SPR wavelength slightly back to longer wavelength $(513 \mathrm{~nm})$ with its intensity maintained, as shown in Fig. 1b. More pronounced changes in this stage are the increase of absorption in the longer

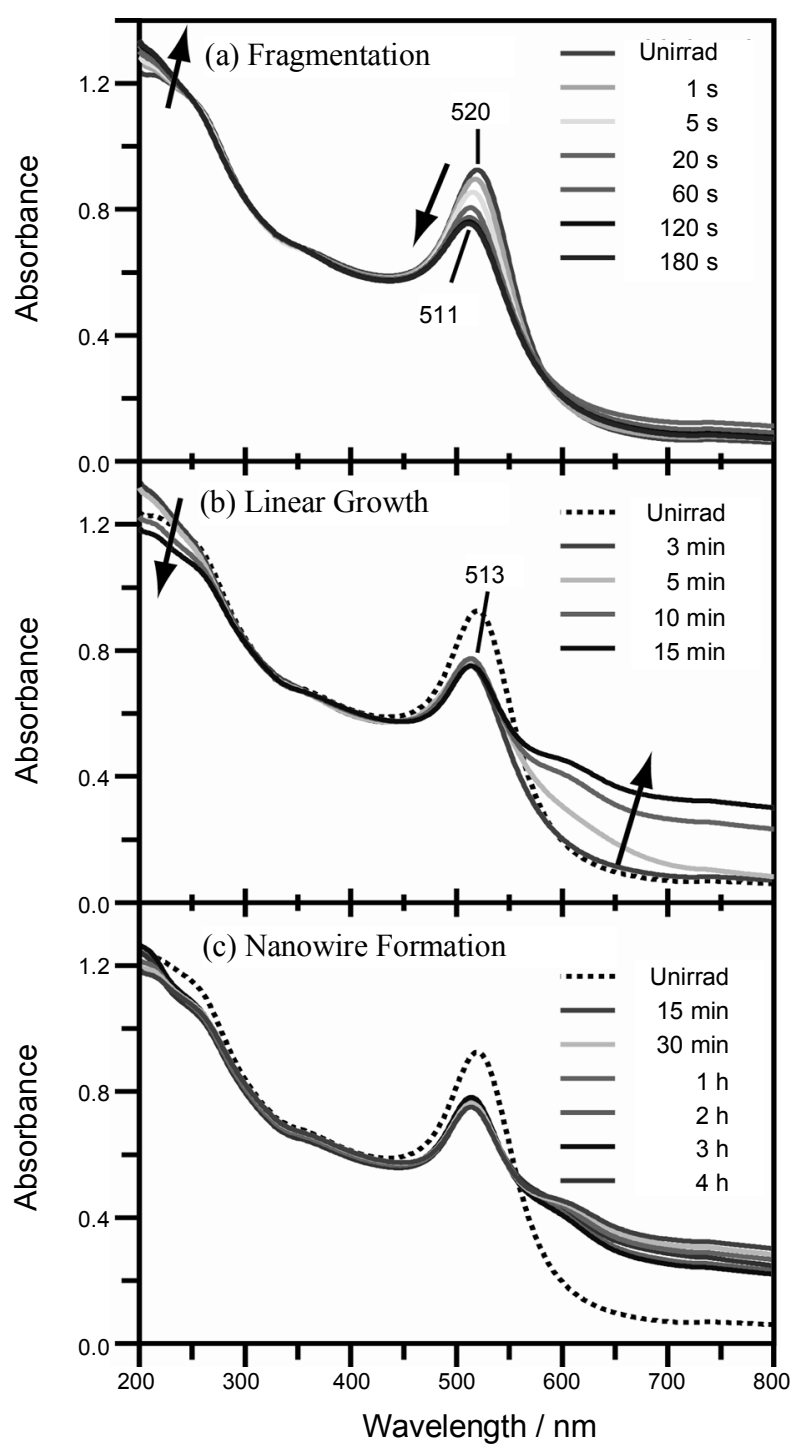

Figure 1. UV-visible absorption spectra of AuNPs irradiated at $532 \mathrm{~nm}$ for indicated periods of time. The evolution is divided into three stages according to spectral features and attributed to (a) fragmentation, (b) linear growth, and (c) complete formation of gold nanowires. The spectra of the original AuNPs before subject to irradiation, designated as "Unirrad", are presented for comparison. The arrows are visual guides to show the direction of the spectral changes with the irradiation time.

wavelength region (>600 nm) and the concomitant decrease of absorption at $\sim 200 \mathrm{~nm}$. Fig. 1c shows that the spectral features remain largely unchanged as we extend the irradiation time up to $4 \mathrm{~h}$.

The absorption of AuNPs near $520 \mathrm{~nm}$ is attributed to SPR. The SPR varies with the size and the shape of gold nanostructures. ${ }^{2,3}$ For example, the SPR band blue-shifts as the size of the AuNPs decreases. AuNPs smaller than $2 \mathrm{~nm}$ do not exhibit SPR. ${ }^{38}$ Furthermore, it has been reported that elongated gold nanostructures such as nanorods or nanowires absorb at $\sim 520$ $\mathrm{nm}$ due to the transverse mode of SPR and additionally at longer wavelengths arising from the longitudinal mode of SPR. ${ }^{39,40}$ Therefore, the rise in absorption at $800 \mathrm{~nm}$ indicates the formation of elongated structures. The absorption at $200 \mathrm{~nm}$ is attributed to gold atoms and small gold clusters with a size less than 

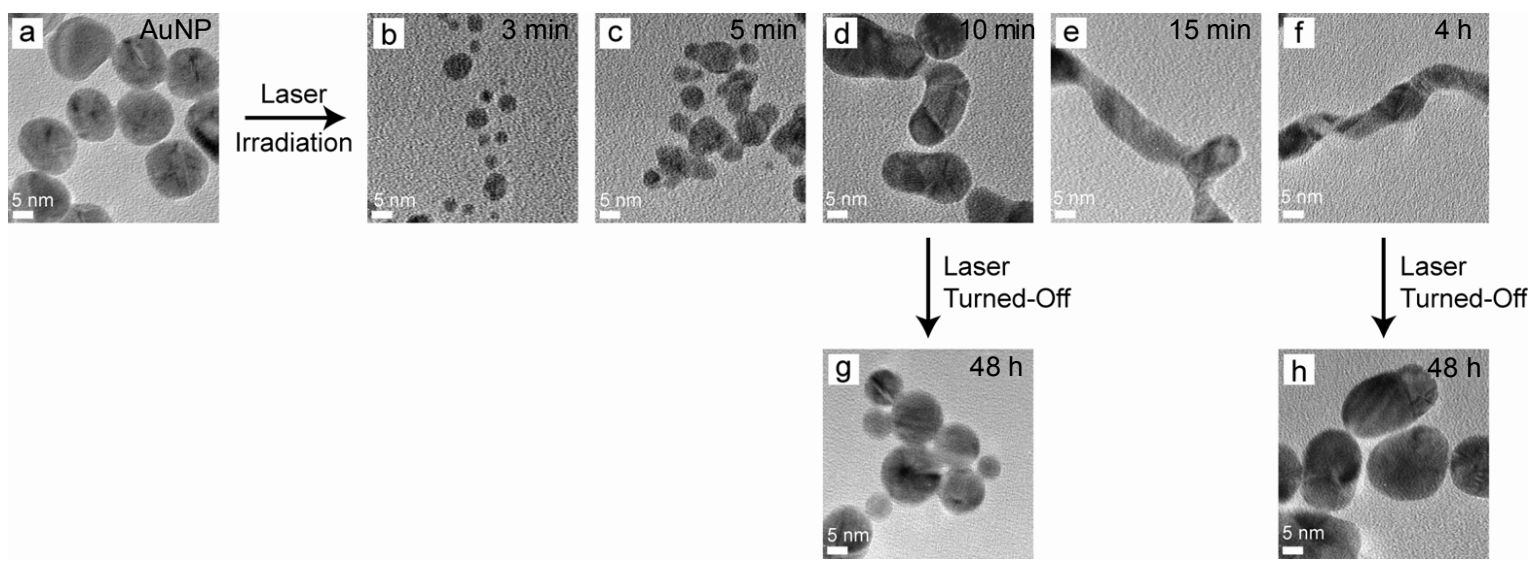

Figure 2. TEM images of AuNPs (a) before irradiation and (b)-(f) after irradiation at $532 \mathrm{~nm}$ for the designated periods of time. Also shown are the images of AuNPs left for $48 \mathrm{~h}$ with the laser turned-off after the irradiation for (g) $10 \mathrm{~min}$ and $(\mathrm{h}) 4 \mathrm{~h}$.

\section{$2 \mathrm{~nm}^{30}$}

Based on the spectral assignments described above, we can deduce morphological changes of AuNPs upon irradiation at $532 \mathrm{~nm}$. For the first $3 \mathrm{~min}$, AuNPs are fragmented into small AuNPs and crumbled gold particles (atoms and clusters). The generation of the former and the latter is reflected in the absorption spectra as the blue shift of the SPR band and the rise in the absorption at $200 \mathrm{~nm}$, respectively. Then, upon irradiation for $5 \mathrm{~min}$, it appears that the size of the small fragmented AuNPs slightly increases as indicated by the slight red shift of SPR (from $511 \mathrm{~nm}$ to $513 \mathrm{~nm}$ ). Further irradiation past 5 min causes a linear growth of AuNPs by consuming gold atoms and clusters, shown as the rise in the absorption at $800 \mathrm{~nm}$ and the concomitant fall at $200 \mathrm{~nm}$ in Fig. 1b. Broad absorption at longer wavelengths suggests that the produced elongated gold nanostructures have a wide distribution of lengths.

The morphological changes are confirmed by the TEM measurements. The TEM images in Fig. 2 obtained after the irradiation for selected periods of time show that AuNPs evolve sequentially from spherical nanoparticles to fragmented smaller nanospheres (3 $\mathrm{min})$, slightly larger nanospheres ( $5 \mathrm{~min}$ ), peanut-shaped nanoparticles (10 $\mathrm{min})$, and, finally, nanowires (15 min and $4 \mathrm{~h}$ ), as we increase the laser irradiation time. The initially prepared AuNPs with a diameter of $16 \pm 1 \mathrm{~nm}$ are fragmented to smaller nanospheres $(4 \pm 2 \mathrm{~nm})$ by the 3 -min irradiation. Apparently gold atoms and clusters produced together, responsible for the rise in absorption in the UV region, are beyond the detection limit of TEM and hence invisible in the image. The size of the fragmented AuNPs grows slightly by further irradiation up to $5 \mathrm{~min}$ (Fig. 2c), consistent with the slight red shift of the SPR band from $511 \mathrm{~nm}$ to $513 \mathrm{~nm}$ in Fig. 1b. As we extend the irradiation time, the AuNPs begin to merge together to form peanut-shaped nanoparticles (nanopeanuts), shown in Fig. $2 d$. Irradiation for 15 min linearly fuses AuNPs further more, leading to the formation of AuNWs. A small magnification view in TEM shows that the AuNWs thus formed are not uniform; the length of the nanowires varies from $\sim 40 \mathrm{~nm}$ to $\sim 80 \mathrm{~nm}$, explaining the broad absorption band assigned to the longitudinal SPR mode of the AuNWs in the UV-visible spectra (Supporting Information).
It appears that the concentration of the stabilizng citrate and laser fluence play a crucial role in the evolution of AuNPs, in agreement with the observation by Kondow and coworkers. 31,41 When we added an extra amount of citrate $(1 \mathrm{mM})$ to the purified AuNP solution before the irradiation, we found that only AuNP fragments were formed by the irradiation while it was AuNWs that were formed by the same period of irradiation without the addition of citrate (Supporting Information). A sufficient amount of citrate present in the solution quickly adsorbs on the fragmented AuNPs and stabilize them. Then, the electrostatic repulsion caused by multilayers of negative citrate anions on the fragmented AuNPs keeps the nanoparticles from approaching and being fused together to form Au nanopeanuts and AuNWs.

The laser irradiation power determines the rate of evolution. When we increased laser power to $700 \mathrm{~mW}$, the complete fragmentation occurred faster ( $1 \mathrm{~min}$ compared to $3 \mathrm{~min}$ at $500 \mathrm{~mW}$ ) and more extensive network of AuNWs was formed by the 30min irradiation. When $300 \mathrm{~mW}$ was used, the irradiation for 30 min yielded only Au nanopeanuts, instead of AuNWs (Supporting Information). No changes in morphology have been observed for the laser power below $200 \mathrm{~mW}$.

The morphological properties of Au nanopeanuts and AuNWs. We investigate the morphological properties of laserproduced Au nanopeanuts and AuNWs. The HRTEM image in Fig. 3 shows the dense solid and crystalline nature of nanopeanuts. Several types of lattice planes with different interplane distances are observed. ${ }^{42}$ In particular, the lattice planes near the border line $(\mathrm{d}=2.20 \AA)$ do not belong to any of the fcc crystals, suggesting that the two particles are merged by fusion that distorts the lattice planes along the merging plane. The crystal facet analysis also suggests that the interparticle fusion of Au nanoparticles occurs rather in random orientation. If the direction of fusion is governed by surface energies, the outer surfaces should be (110) or (100) with high surface energies that are stabilized by citrate and thus the fusion should occur specifically in the (111) direction, which clearly is not the case. ${ }^{43}$ Therefore, it appears that random collisions among nanoparticles, followed by instantaneous fusion by laser heating produce the peanut-shaped nanoparticles.

The Au nanopeanuts are further combined to form AuNWs 


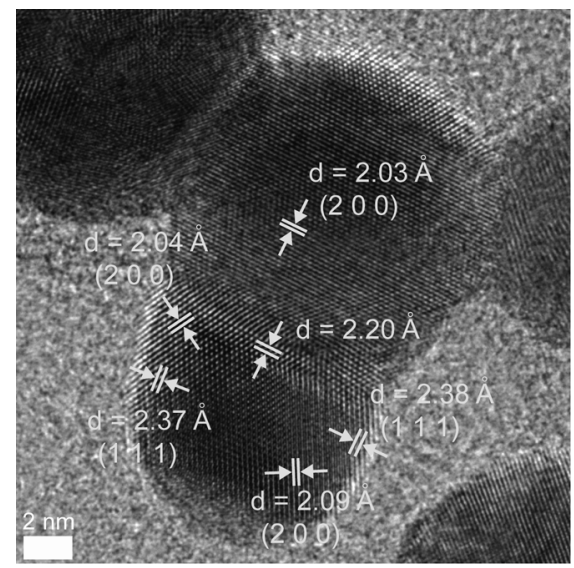

Figure 3. An HRTEM image of an Au nanopeanut prepared by irradiation of AuNPs for $10 \mathrm{~min}$.

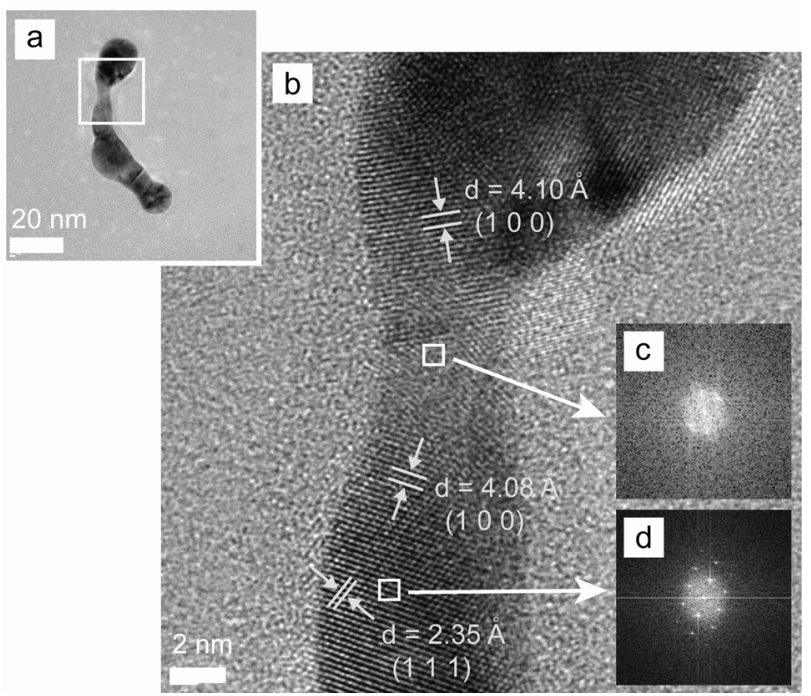

Figure 4. (a) A TEM image of the AuNW prepared by irradiation of AuNPs for 15 min. (b) An HRTEM image of a part of the AuNW marked by the square in (a). Crystal facets assigned by the distances between lattice planes are included. (c) and (d) are fast Fourier transform images of the bridging junction area and the crystalline area of the AuNW, respectively.

by laser irradiation. Fig. 4 shows the HRTEM image of an AuNW. The crystalline nanoparticles are bridged by noncrystalline, fluffy solids. The fast Fourier transform image of the junction of the AuNW, looking fuzzy and lacking bright diffraction spots, clearly shows the amorphous nature of the region in contrast to the crystalline part of the AuNW (Figs. 4c and 4d). The amorphous noncrystalline accumulation of gold atoms is probably one of the reasons for the facile disintegration of the AuNWs in the absence of laser pulses (vide infra). Once the laser is turned off, gold atoms slowly diffuse away from the amorphous region and adsorb on the thermodynamically favored crystalline surfaces (Ostwald ripening), leading to the cleavage of AuNWs into spherical AuNPs. Such intraparticle ripening is also corroborated by the larger diameter of the recovered nanospheres than the thickness of the AuNWs as observed in Fig. 2. (a) Au Nanopeanuts

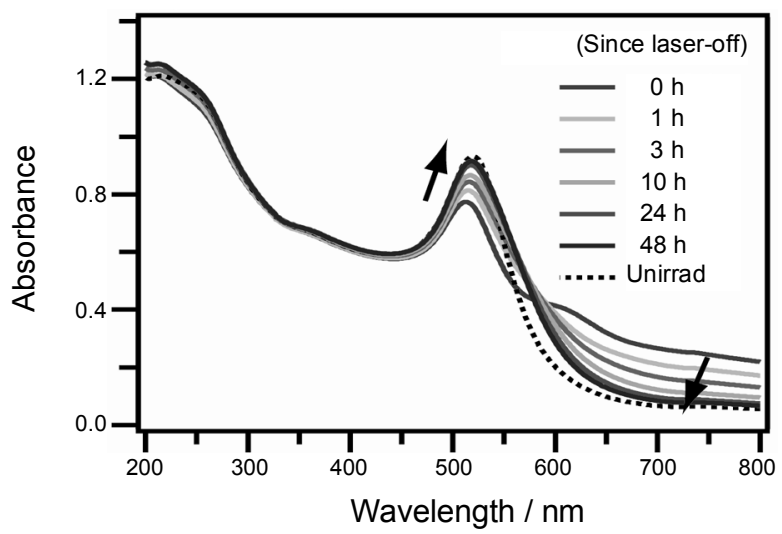

(b) Au Nanowires

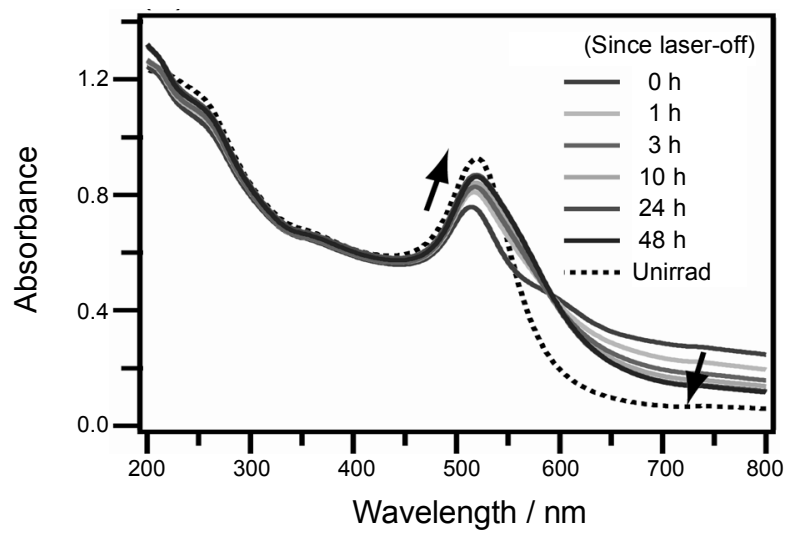

Figure 5. The change of UV-visible absorption spectra of (a) Au nanopeanuts and (b) AuNWs as time elapses after the termination of the laser irradiation. Au nanopeanuts and AuNWs are prepared by irradiation of AuNPs for $10 \mathrm{~min}$ and $4 \mathrm{~h}$, respectively, as described in the text. The spectra of the unirradiated AuNPs (dotted lines) are presented for comparison. The arrows are visual guides to show the direction of the spectral changes with the elapsed time.

The stability of Au nanopeanuts and AuNWs. Figs. 2d and 3 show that the particles are fused together to form Au nanopeanuts, not just assembled by weak interparticle forces. Furthermore, the AuNWs endure the continuing laser irradiation at least for $4 \mathrm{~h}$ without refragmentation. These observations led us to believe that the Au nanopeanuts and AuNWs produced by the laser irradiation are stable and robust. On the contrary to the expectation, however, we observed that both Au nanopeanuts and AuNWs slowly disintegrate, restoring the spherical AuNPs when the laser irradiation discontinues. Fig. 5 shows that the UV-visible spectra of Au nanopeanuts and AuNWs slowly recovers those of Au nanospheres as time elpases after the termination of laser irradiation. The decrease in absorption in the long wavelength region and the simultaneous increase in absorption at $520 \mathrm{~nm}$ clearly indicate that the elongated parts of nanopeanuts and nanowires spontaneously dissolve, reshaping into spheres over $48 \mathrm{~h}$. The TEM images in Figs. $2 \mathrm{~g}$ and $2 \mathrm{~h}$ confirm the transformation.

As mentioned earlier, it seems that once the laser irradiation is stopped, the one-dimensional structures dissolve into the thermodynamically stable spherical shape. In particular, when the 


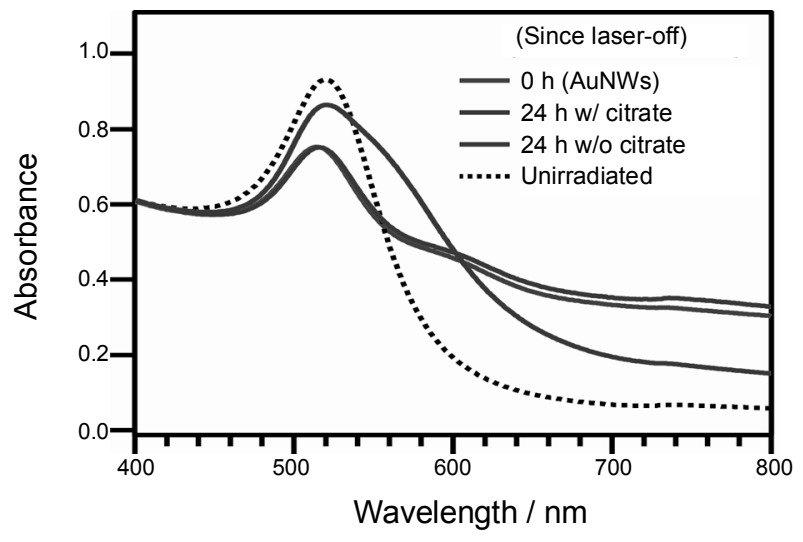

Figure 6. Stabilization of AuNWs by the addition of citrate. The $\mathrm{Au}-$ NWs prepared by irradiation of the AuNPs for 30 min (red line) transform back to the spherical AuNPs when left idle for $24 \mathrm{~h}$ without further laser irradiation (blue line). The addition of citrate solution to $\mathrm{Au}-$ NWs stabilizes the AuNWs (green line).

surfaces of Au nanopeanuts and AuNWs are not stabilized enough by citrate due to the limited amount available, or when they do not possess well-developed crystal facets, they would slowly transform into spheres to reduce the overall surface energies.

Indeed, the disintegration of the laser-produced Au nanopeanuts and AuNWs into nanospheres can be effecitvely prevented by the addition of extra amount of citrate. We add a citrate solution $(1 \mathrm{mM})$ after producing AuNWs by 30 min of laser irradiation, and see if the AuNWs remain unchanged when the irradiation is stopped. Fig. 6 shows that the AuNWs remain stable when citrate is added while the AuNWs without the addition of citrate slowly transform back to the spherical AuNPs in $24 \mathrm{~h}$. A sufficient amount of capping ligands stabilizes the surfaces of Au nanopeanuts and AuNWs and inhibits further structural changes.

\section{Conclusions}

In this paper, we have shown how irradiation of AuNPs with a laser $\left(532 \mathrm{~nm}, \sim 8 \mathrm{~ns}, 130 \mathrm{~mJ} / \mathrm{cm}^{2} \cdot\right.$ pulse, $\left.10 \mathrm{~Hz}\right)$ leads to the formation of nanopeanuts and nanowires. We also have revealed that the photoproducts (nanopeanuts, nanowires) transform back to the spherical shape when the laser irradiation stops. The changes in the UV-visible absorption wavelengths and intensities allowed us to follow the collective and synchronous movement of particles under laser irradiation. The TEM measurements confirmed the morphological changes of AuNPs. The HRTEM images showed that the interparticle fusion occurs in random orientation for the formation of Au nanopeanuts and some junctions of AuNWs are amorphous. We found that citrate is effective in stabilizing the AuNWs.

Acknowledgments. This research was supported by Basic Science Research Program through the National Research Foundation of Korea (NRF) funded by the Ministry of Education, Science and Technology (KRF-2007-314-C00147). This research was also supported by the Graduate Research Assistantship of Dankook University in 2009.
Supporting Information available. Included are details of UV-visible spectra of synthesized and purified AuNPs, a wide view of a TEM image of the AuNWs prepared by irradiation, and dependence of evolution of AuNPs on the concentration of citrate and laser fluence.

\section{References}

1. Jain, P. K.; Huang, X.; El-Sayed, I. H.; El-Sayed, M. A. Acc. Chem. Res. 2008, 41, 1578.

2. Jain, P. K.; Lee, K. S.; El-Sayed, M. A. J. Phys. Chem. B 2006, 110, 7238.

3. Kelly, K. L.; Coronado, E.; Zhao, L. L.; Schatz, G. C. J. Phys. Chem. B 2003, 107, 668.

4. Hirsch, L. R.; Stafford, R. J.; Bankson, J. A.; Sershen, S. R.; Rivera, B.; Price, R. E.; Hazle, J. D.; Halas, N. J.; West, J. L. Proc. Natl. Acad. Sci. U.S.A. 2003, 100, 13549.

5. Huang, W.; Qian, W.; El-Sayed, M. A. J. Am. Chem. Soc. 2006, $128,13330$.

6. El-Sayed, I. H.; Huang, X.; El-Sayed, M. A. Cancer Lett. 2006, 239, 129.

7. O'Neal, D. P.; Hirsch, L. R.; Halas, N. J.; Payne, J. D.; West, J. L. Cancer Lett. 2004, 209, 171.

8. Loo, C. A.; Lowery, A.; Halas, N.; West, J.; Drezek, R. Nano Lett. 2005, 5, 709 .

9. Wang, Y.; Xie, X.; Wang, X.; Ku, G.; Gill, K. L.; O'Neal, D. P.; Stoica, G.; Wang, L. V. Nano Lett. 2004, 4, 1689.

10. Gobin, A. M.; Lee, M. H.; Halas, N. J.; James, W. D.; Drezek, R. A.; West, J. L. Nano Lett. 2007, 7, 1929.

11. Loo, C.; Hirsch, L.; Lee, M. H.; Chang, E.; West, J.; Halas, N.; Drezek, R. Opt. Lett. 2005, 30, 1012.

12. Camden, J. P.; Dieringer, J. A.; Zhao, J.; Van Duyne, R. P. Acc. Chem. Res. 2008, 41, 1653.

13. Moskovits, M. Rev. Mod. Phys. 1985, 57, 783.

14. Nie, S.; Emory, S. R. Science 1997, 275, 1102.

15. Kneipp, K.; Wang, Y.; Kneipp, H.; Perelman, L. T.; Itzkan, I.; Dasari, R. R.; Feld, M. S. Phys. Rev. Lett. 1997, 78, 1667.

16. Cao, Y. C.; Jin, R.; Mirkin, C. A. Science 2002, 297, 1536.

17. Burda, C.; Chen, X.; Narayanan, R.; El-Sayed, M. A. Chem. Rev. 2005, 105, 1025 .

18. Daniel, M.-C.; Astruc, D. Chem. Rev. 2004, 104, 293.

19. Wang, Z. L.; Petroski, J. M.; Green, T. C.; El-Sayed, M. A. J. Phys. Chem. B 1998, 102, 6145 .

20. Jana, N. R.; Gearheart, L.; Murphy, C. J. J. Phys. Chem. B 2001, 105, 4065.

21. Skrabalak, S. E.; Chen, J.; Sun, Y.; Lu, X.; Au, L.; Cobley, C. M.; Xia, Y. Acc. Chem. Res. 2008, 41, 1587.

22. Bae, Y.; Kim, N. H.; Kim, M.; Lee, K. Y.; Han, S. W. J. Am. Chem. Soc. 2008, 130, 5432 .

23. Lee, J.; Hasan, W.; Stender, C. L.; Odom, T. W. Acc. Chem. Res. 2008, 41, 1762.

24. Duffus, C.; Camp, P. J.; Alexander, A. J. J. Am. Chem. Soc. 2009, $131,11676$.

25. Klajn, R.; Wesson, P. J.; Bishop, K. J. M.; Grzybowski, B. A. Angew. Chem., Int. Ed. 2009, 48, 1.

26. Link, S.; Burda, C.; Mohamed, M. B.; Nikoobakht, B.; El-Sayed, M. A. J. Phys. Chem. A 1999, 103, 1165.

27. Link, S.; Burda, C.; Nikoobakht, B.; El-Sayed, M. A. J. Phys. Chem. B 2000, 104, 6152.

28. Kurita, H.; Takami, A.; Koda, S. Appl. Phys. Lett. 1998, 72, 789.

29. Mafuné, F.; Kohno, J.-y.; Takeda, Y.; Kondow, T. J. Phys. Chem. B 2002, 106, 7575.

30. Mafuné, F.; Kohno, J.-y.; Takeda, Y.; Kondow, T. J. Phys. Chem. $B$ 2002, 106, 8555 .

31. Mafuné, F.; Kohno, J.-y.; Takeda, Y.; Kondow, T. J. Phys. Chem. B 2003, 107, 12589. 
32. Takami, A.; Kurita, H.; Koda, S. J. Phys. Chem. B 1999, 103, 1226.

33. Ahmadi, T. S.; Logunov, S. L.; El-Sayed, M. A. J. Phys. Chem. 1996, 100,8053

34. Hodak, J. K.; Martini, I.; Hartland, G. V. J. Phys. Chem. B 1998, 102,6958

35. Perner, M.; Bost, P.; Lemmer, U.; Plessen, G. v.; Feldmann, J.; Becker, U.; Mennig, M.; Schmitt, M.; Schmidt, H. Phys. Rev. Lett. 1997, 78, 2192.

36. Peng, Z.; Walther, T.; Kleinermanns, K. J. Phys. Chem. B 2005, 109, 15735.

37. Turkevich, J.; Stevenson, P. C.; Hillier, J. Discuss. Faraday Soc.
1951, 11, 55 .

38. Alvarez, M. M.; Khoury, J. T.; Schaaff, T. G.; Shafigullin, M. N.; Vezmar, I.; Whetten, R. L. J. Phys. Chem. B 1997, 101, 3706.

39. Murphy, C. J.; Sau, T. K.; Gole, A. M.; Orendorff, C. J.; Gao, J.; Gou, L.; Hunyadi, S. E.; Li, T. J. Phys. Chem. B 2005, 109, 13857.

40. Link, S.; Mohamed, M. B.; El-Sayed, M. A. J. Phys. Chem. B 1999, $103,3073$.

41. Mafuné, F.; Kohno, J.-y.; Takeda, Y.; Kondow, T. J. Phys. Chem. B 2001, 105, 9050.

42. JCPDS database (04-0784, 02-1095, or 01-1174)

43. Lofton, C.; Sigmund, W. Adv. Funct. Mater. 2005, 15, 1197. 